\title{
Changes in visual function and thickness of macula after photodynamic therapy for age-related macular degeneration
}

This article was published in the following Dove Press journal:

Clinical Ophthalmology

3I August 2009

Number of times this article has been viewed

\author{
Kyoko Okada \\ Mariko Kubota-Taniai \\ Masayasu Kitahashi \\ Takayuki Baba \\ Yoshinori Mitamura \\ Shuichi Yamamoto \\ Department of Ophthalmology \\ and Visual Science, Chiba University \\ Graduate School of Medicine, Chiba, \\ Japan
}

Purpose: To determine the correlation between the changes in the central retinal sensitivity and the changes in the foveal thickness (FT) after photodynamic therapy (PDT) for age-related macular degeneration (AMD).

Methods: Nineteen eyes of 19 patients with choroidal neovasularizations (CNVs) secondary to AMD were studied. The pretreatment values of the central retinal sensitivity determined by Micro Perimeter 1 (MP1; Nidek Technologies), best-corrected visual acuity (BCVA), and optical coherence tomography (OCT)-determined FT were compared to the postoperative values at three and six months after PDT.

Results: At six months, the retinal sensitivity within the central $10^{\circ}$ was significantly improved $(P=0.02)$ and the FT was significantly thinner $(P=0.016)$. The BCVA, however, did not change significantly $(P=0.80)$. The changes in the retinal sensitivities were significantly correlated with the changes in the decrease in the FT $\left(\mathrm{r}=-0.59, P=0.012\right.$ within the central $\left.10^{\circ}\right)$ at six months after PDT.

Conclusion: Significant improvements in retinal sensitivities within the central $10^{\circ}$ and a decrease in FT were observed even though the BCVA was not significantly improved. The measurement of retinal sensitivity by MP1 may be a better method to assess central visual function than the conventional visual acuity after PDT.

Keywords: age-related macular degeneration, fundus-related microperimetry, optical coherence tomography, photodynamic therapy

\section{Introduction}

Photodynamic therapy (PDT) with verteporfin has been shown to reduce the degree of vision decrease in patients with a subfoveal choroidal neovascularization (CNV) secondary to age-related macular degeneration (AMD) and polypoidal choroidal vasculopathy (PCV). ${ }^{1-4}$ PDT also results in a resolution of exudative and/or hemorrhagic lesions leading to a reduction of foveal thickness (FT) measured by optical coherence tomography (OCT). ${ }^{5,6}$

The distance visual acuity (VA) is the standard method of assessing the central visual function that is used routinely in the clinic. However, patients are often encountered who state that their central vision has improved and ophthalmoscopy showed a reduction of the exudation after the PDT, even though their conventional VA was not improved. These cases suggested that VA does not necessarily measure the visual quality, and may not represent the practical foveal or macular function.

It has been established that fundus-related perimetry can be used to measure macular function. ${ }^{7-9}$ Micro Perimeter 1 (MP1; Nidek Technologies, Gamagori, Japan) is a
Correspondence: Kyoko Okada Department of Ophthalmology and Visual Science, Chiba University

Graduate School of Medicine,

I-8-I Inohana, Chuo-ku,

Chiba 2608670, Japan

$\mathrm{Tel}+8 \mid 432262123$

Fax +81432271810

Email okada-k@umin.net 
recently introduced fundus-related microperimetry, which is used to obtain quantitative and reliable measurements of retinal sensitivity which corrects for eye movements during the measurements. This allows for more accurate measurements of the central retinal sensitivities even in patients with unstable fixation, and the perimetric findings from MP1 are comparable to those obtained by scanning laser ophthalmoscopy (SLO). ${ }^{10}$ The software programs of the MP1 can superimpose the retinal sensitivities on digital fundus images. In eyes with PCV, the retinal sensitivity measured by MP1 has been shown to improve shortly after PDT. ${ }^{11}$

The purpose of this study was to determine the central retinal sensitivities after PDT in eyes with AMD, and to correlate the changes in the central retinal sensitivities with the changes in the best-corrected VA (BCVA) and with the FT measured by OCT after PDT.

\section{Materials and methods}

Nineteen eyes of 19 patients that underwent PDT as an initial treatment for $\mathrm{CNV}$ secondary to AMD were studied. Of the 19 eyes, five had predominantly classic CNV, three had occult CNV with no signs of classic CNV, one had minimally classic CNV, and 10 had PCV. Eyes that underwent a second PDT within six months, and eyes that had other medical treatments for AMD, eg, transpupillary thermotherapy and macular photocoagulation, were excluded. The age of the patients ranged from 57 to 91 years with a mean of $71.4 \pm 7.3$ years $( \pm \mathrm{SD})$.

The procedures used conformed to the tenets of the Declaration of Helsinki, and informed consent was obtained from all subjects after the nature and possible consequences of the study were explained.

Fundus-related microperimetry was performed with MP1 using the software available for the 2006 model (Version: MP1 SW 1.4.2.SP1; Nidek Technologies) with automated corrections for eye movements. The follow-up examinations used a program which automatically selects the retinal sites that are exactly over the previously tested retinal points.

The auto-tracking system calculates the $\mathrm{x}$ and $\mathrm{y}$ positions, and the rotational shifts relative to a reference frame obtained by an infrared camera at the beginning of the examination. The Goldmann III stimulus with a 4-2 staircase strategy was used, and a rectangular $3^{\circ}$ test grid with 24 stimulus locations covering an area of $10^{\circ}$ was examined (Figure 1). The stimuli were projected on a white background with a luminance of $1.27 \mathrm{~cd} / \mathrm{m}^{2}$ and a stimulus duration of $100 \mathrm{msec}$. The differential luminance, defined as the difference between stimulus luminance and background luminance,

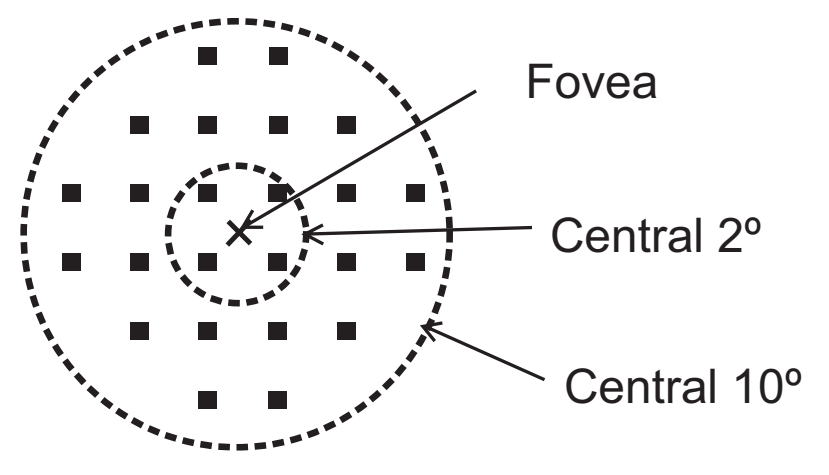

Figure I Results of microperimetry. Twenty-four loci covering the central $10^{\circ}$ were examined by Micro Perimeter I (MPI). Four measurement points were located within the central $2^{\circ}$ of the macula, and 24 measurement points were located within the central $10^{\circ}$ of the macula.

was $127 \mathrm{~cd} / \mathrm{m}^{2}$ at $0 \mathrm{~dB}$ stimulation, and the maximum stimulus attenuation was $20 \mathrm{~dB}$.

PDT with verteporphin was performed with the standard protocol. MP1 examinations and other comprehensive ophthalmic examinations including measurement of BCVA, OCT, and indirect ophthalmoscopy were performed before treatment, and at three and six months after PDT. Fluorescein angiography (FA) and indocyanine green angiography (ICGA) were performed on all patients before and at three months after PDT.

From the FA/ICGA findings at three months after PDT, eyes were classified as those with "no leakage" from the CNV, "minor leakage", and "progression" group, ie, leakage extending beyond the area of the lesion identified at the baseline.

The BCVA was measured with a standard Landolt $\mathrm{C}$ chart, and the values were converted to the logarithm of the minimal angle of resolution ( $\log$ MAR) units. The FT was defined as the distance between the vitreoretinal interface and the retinal pigment epithelium in the center of the fovea using the Stratus OCT3000 (Carl Zeiss Meditec, Dublin, CA, USA).

Patients who stated that they had a definite improvement in their central visual disturbance at six months after PDT were grouped in the Yes group, and patients who stated that they had no changes or had a decrease in their central visual disturbance were grouped together in the Other group.

The numerical data were analyzed by the Wilcoxon signed-rank test. Bivariate relationships were assessed using the Spearman's correlation test. A $P$ value of $<0.05$ was considered statistically significant. The changes in retinal sensitivity were defined as the difference in retinal sensitivity $(\mathrm{dB})$ at baseline (pre-treatment) from the posttreatment retinal sensitivity in $\mathrm{dB}$. The changes in the FT were defined as the ratio of the FT at baseline divided by 
the post-treatment FT. Changes in the BCVA were defined as the difference of the BCVA in $\log$ MAR units at baseline subtracted from the post treatment logMAR BCVA.

\section{Results}

The fundus photographs, OCT images, and microperimetric results before, and at three and six months after PDT obtained from a 61-year-old man are shown in Figure 2. His BCVA improved slightly from 20/60 to 20/50 at six months after PDT, and the mean retinal sensitivity within the central $10^{\circ}$ improved from $5.0 \mathrm{~dB}$ at baseline to $9.1 \mathrm{~dB}$ at three months and to $11.8 \mathrm{~dB}$ at six months after PDT.

The mean changes in the best-corrected BCVA in logMAR units, FT, and retinal sensitivities from baseline at three and six months after PDT are presented in Table 1. Although the
FT was reduced significantly after PDT at both three and six months ( $P=0.0015$ and $P=0.016$, respectively), the changes in BCVA from baseline was not statistically significant. The improvement in the mean retinal sensitivities within the central $10^{\circ}$ was statistically significant at six months $(P=0.02)$, but not statistically significant within the central $2^{\circ}(P=0.39$ at three months and $P=0.11$ at six months $)$.

The relationship between the changes in retinal sensitivity from the baseline after PDT and changes from baseline in the BCVA or FT after PDT are shown in the scatter diagrams (Figures 3-5). The changes in the BCVA was significantly correlated with the changes in FT at six months after PDT ( $\mathrm{r}=0.51 ; P=0.03$ ), and not significantly correlated with the changes in FT at three months after PDT $(r=0.46$; $P=0.05$ ) (Figure 3 ). The changes in retinal sensitivities

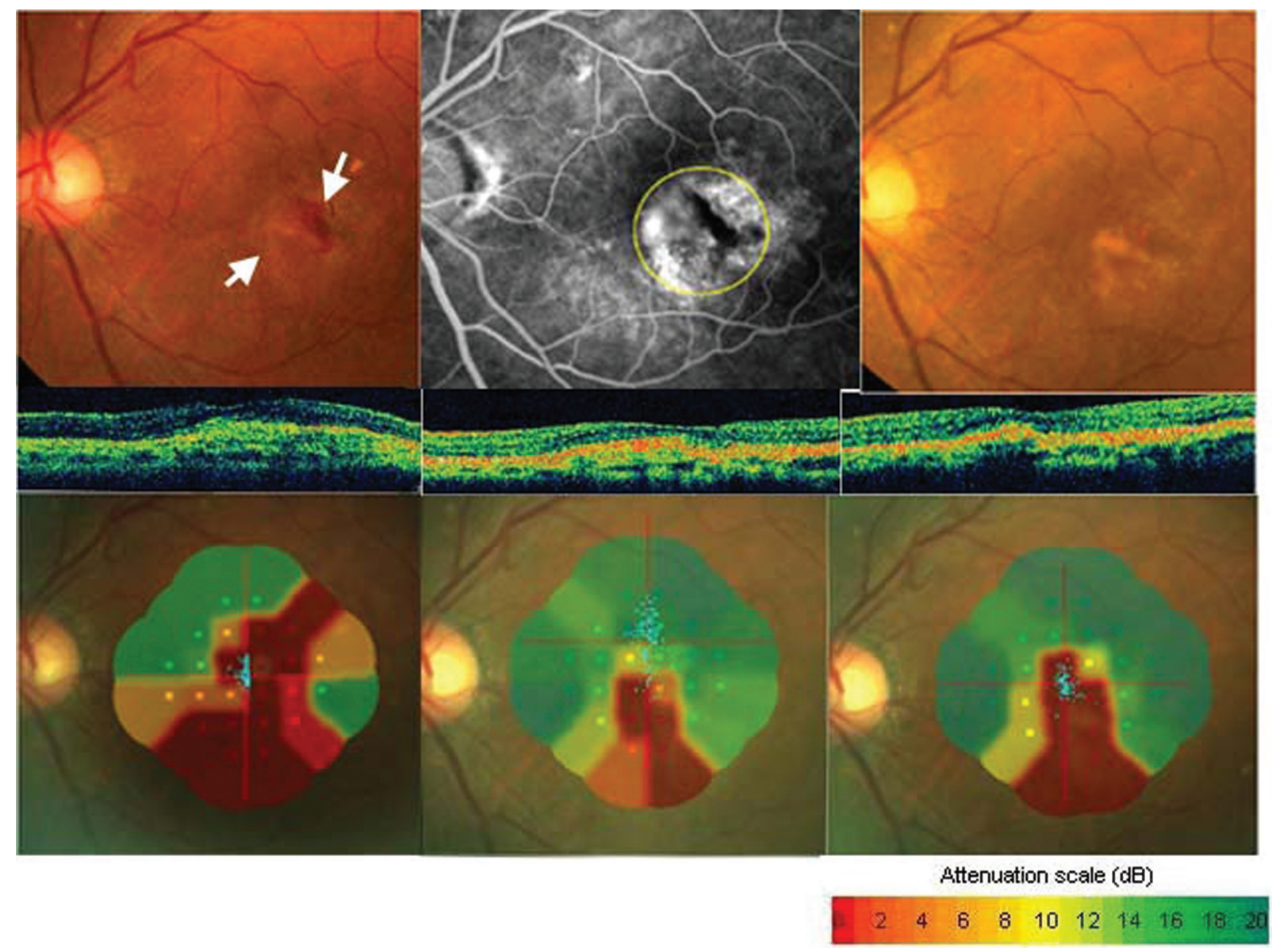

Figure 2 Fundus photographs, fluorescein angiograms, optical coherence tomographic (OCT) images, and Micro Perimeter I (MPI) images from a 6 I-year-old man (Case I) whose visual acuity (VA) was reduced to $20 / 60$ secondary to classic and occult choroidal neovascularization (CNV) in his left eye. Top left: Fundus photograph showing subretinal hemorrhage and classic CNV (white arrow) before treatment. Top middle: Fluorescein angiogram (FA) showing minimally classic CNV. Photodynamic therapy was performed with a $3500 \mathrm{im}$ laser spot on the area indicated by the yellow dotted circle. Top right: Fundus photograph three months after photodynamic therapy (PDT) shows fibrosis of CNV and absorption of subretinal hemorrhage. VA was 20/50 at three and six months after PDT. Middle row, left: OCT image before PDT. Middle row, middle: OCT image three months after PDT showing a reduction in the size of the CNV. Middle row, right: OCT image at six months after PDT showing a reduction of foveal thickness. Bottom left: MPI image shows a dense scotoma (not seen at $0 \mathrm{~dB}$ ) in CNV component and subretinal hemorrhage. Mean retinal sensitivity within the central I0 is $5.0 \mathrm{~dB}$ before PDT. Bottom middle: Mean retinal sensitivity improved to $9.1 \mathrm{~dB}$ at three months after PDT. Bottom right: Mean retinal sensitivity improved to II.8 dB at six months after PDT. The patient did not report an improvement of the central visual disturbance of the left eyes. FA shows no leakage from CNV at three months after PDT. 
Table I Changes in visual acuity, foveal thickness, and mean retinal sensitivity

\begin{tabular}{|c|c|c|c|}
\hline & Baseline & Month 3 & Month 6 \\
\hline Visual acuity (logMAR) & $0.61 \pm 0.34$ & $\begin{array}{l}0.64 \pm 0.47 \\
(P=0.91)\end{array}$ & $\begin{array}{l}0.65 \pm 0.48 \\
(P=0.80)\end{array}$ \\
\hline Foveal thickness $(\mu \mathrm{m})$ & $407 \pm 144$ & $\begin{array}{l}253 \pm 118 \\
\left(P=0.0015^{*}\right)\end{array}$ & $\begin{array}{l}286 \pm 153 \\
(P=0.016 *)\end{array}$ \\
\hline $\begin{array}{l}\text { Mean retinal sensitivity, } \\
2 \operatorname{deg}(\mathrm{dB})\end{array}$ & $1.7 \pm 3.1$ & $\begin{array}{l}2.7 \pm 4.3 \\
(P=0.39)\end{array}$ & $\begin{array}{l}3.3 \pm 4.4 \\
(P=0.1 I)\end{array}$ \\
\hline $\begin{array}{l}\text { Mean retinal sensitivity, } \\
10 \mathrm{deg}(\mathrm{dB})\end{array}$ & $2.8 \pm 3.1$ & $\begin{array}{l}4.1 \pm 3.7 \\
(P=0.098)\end{array}$ & $\begin{array}{l}5.1 \pm 5.0 \\
(P=0.02 *)\end{array}$ \\
\hline
\end{tabular}

Notes: Statistical analyses were performed using Wilcoxon signed-rank test between baseline and month 3 and between baseline and month 6 . ${ }^{*}>0.05$, compared with the pre-treatment value.

was significantly correlated with the changes in FT within the central $2^{\circ}(\mathrm{r}=-0.49 ; P=0.038)$ and within the central $10^{\circ}(\mathrm{r}=-0.59 ; P=0.012)$ at six months after PDT (Figure 4). The correlations between the changes in retinal sensitivity and the BCVA were not significant within the central $2^{\circ}(\mathrm{r}=-0.39 ; P=0.09)$ and within the central $10^{\circ}(\mathrm{r}=-0.39$; $P=0.09$ ) at six months after PDT (Figure 5).

At six months after PDT, 14 patients (74\%) reported that their central vision had improved, however only two of these patients had gained more than $0.2 \log$ MAR units and the mean change in VA was $-0.09 \pm 0.12$ in these patients (Table 2). A comparison of the mean change in retinal sensitivity at six months between patients who reported an improvement and those who did not report an improvement showed that there was a significant improvement in the retinal sensitivity in patients who reported a subjective improvement (Table 2; $P=0.016$ within central $2^{\circ}$ and $P=0.04$ within

(Month 3)

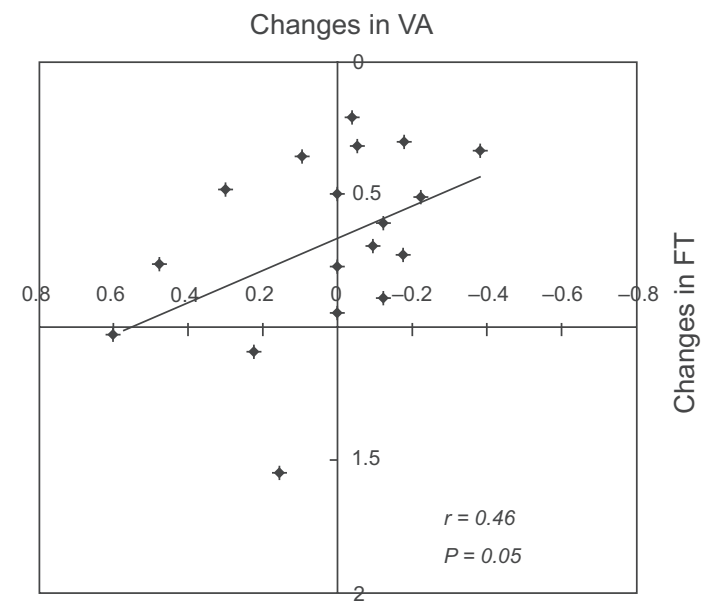

Table 2 Subjective improvement and changes in VA, FT, and retinal sensitivity at six months after PDT

\begin{tabular}{llll}
\hline & \multicolumn{2}{l}{$\begin{array}{l}\text { Improvement of symptomatic } \\
\text { visual disturbance }\end{array}$} & $\mathbf{P}$ \\
\cline { 2 - 3 } & $\begin{array}{l}\text { Yes } \\
(\mathbf{n}=\mathbf{1 4 , 7 4 \% )}\end{array}$ & $\begin{array}{l}\text { Others } \\
(\mathbf{n}=\mathbf{5 , 2 6 \% )}\end{array}$ \\
\hline Changes in VA & $-0.09 \pm 0.12$ & $0.35 \pm 0.18$ & 0.001 \\
Changes in FT $(\mu \mathrm{m})$ & $-182.1 \pm 172.9$ & $49.2 \pm 103.5$ & 0.012 \\
Changes in retinal & & & \\
sensitivity $(\mathrm{dB})$ & & & \\
Within 2 & & & \\
Within $10^{\circ}$ & $2.7 \pm 5.0$ & $-3.5 \pm 4.2$ & 0.016 \\
\hline
\end{tabular}

Notes: Numerical data were analyzed using Mann-Whitney $U$ test.

Abbreviations: CNV, choroidal neovascularization; FT, foveal thickness; PDT, photodynamic therapy; VA, visual acuity.

central $10^{\circ}$; Mann-Whitney $U$ test), as well as those with a reduction in the FT $(P=0.012$; Mann-Whitney $U$ test $)$.

FA showed no leakage from the CNV in 10 eyes (53\%), minor leakage in six eyes (32\%), and progression of the CNV in three (15\%) eyes at three months after PDT. Three patients with $\mathrm{CNV}$ progression refused additional treatments. A comparison of the mean change in retinal sensitivity and VA between eyes with no leakage from CNV and eyes with minor leakage or advancement of the CNV at three months after PDT is shown in Table 3. A significant improvement in retinal sensitivity was observed only in eyes with no leakage from the $\operatorname{CNV}\left(P=0.01\right.$ within central $2^{\circ}$, and $P=0.04$ within central $10^{\circ}$; Mann-Whitney $U$ test).

\section{Discussion}

A significant improvement in the retinal sensitivities within the central $10^{\circ}$ and a significant reduction in the FT were observed

Figure 3 Relationship between changes in best-corrected visual acuity (BCVA) and changes in foveal thickness (FT). There is a significant correlation between these two parameters at six months after photodynamic therapy $(r=0.51 ; P=0.03)$.

(Month 6)

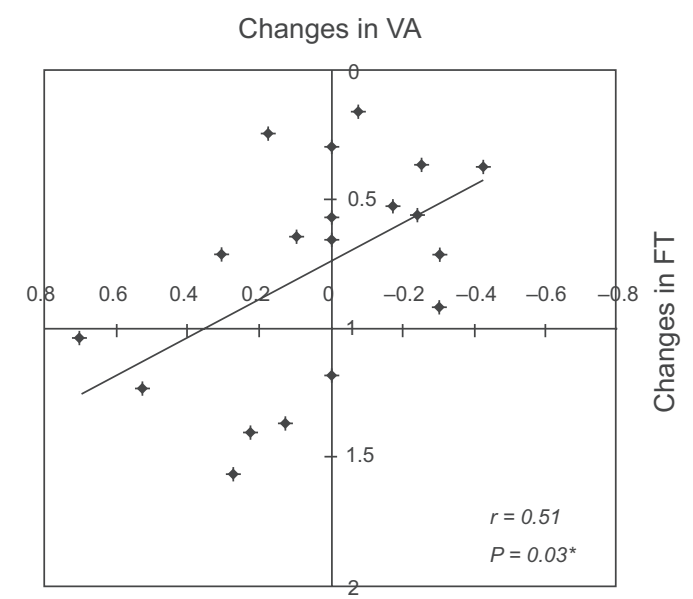



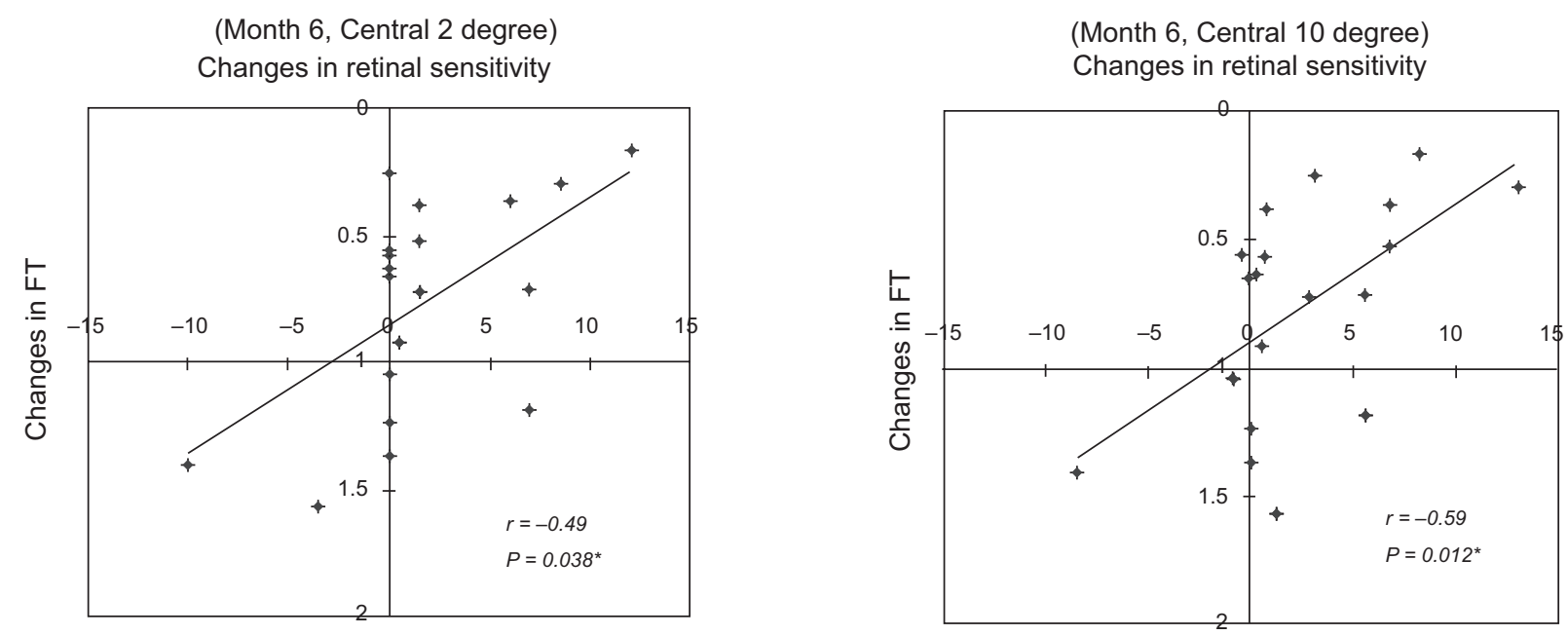

Figure 4 Relationship between changes in retinal sensitivity and the decrease in foveal thickness (FT). The changes in retinal sensitivity were significantly correlated to the changes in foveal thickness. Data from within the central $2^{\circ}(r=-0.49 ; P=0.038)$ and within the central $10^{\circ}(r=-0.59 ; P=0.012)$.

at six months after PDT for CNV due to AMD. However, the conventional BCVA, eg, Snellen visual acuity, in these eyes did not improve significantly, although $74 \%$ of these patients reported a subjective improvement of their central vision. These findings suggested that distance VA may not be a good way to assess the central visual function in eyes with a subfoveal CNV. This is because the CNV may affect the central vision and result in a scotoma which has only a slight influence on distant vision but has a strong effect on near vision..$^{8,12}$

In agreement with this study, we have reported a significant correlation between retinal sensitivity measured with the microperimeter and the VA and the FT in normal patients, and patients with diabetic macular edema. ${ }^{13}$

Eyes with AMD have different manifestations within the central $10^{\circ}$, eg, a CNV, subretinal hemorrhage, subretinal fluid, and a chorioretinal atrophy. The relationship between the retinal sensitivity and anatomic abnormalities based on funduscopic and angiographic findings showed that scotomas (not seen at $0 \mathrm{~dB}$ ) were initially located in the areas corresponding to the classic and/or occult components of the CNV, PCV, chorioretinal atrophy, and subretinal hemorrhages. The relative scotomas were located in the area of neurosensory retinal detachment, consistent with observations of Tezel and colleagues ${ }^{7}$ and Schmidt-Erfurth and colleagues. ${ }^{14}$ After PDT, there was a reduction of the subretinal fluid and subretinal hemorrhage, which led to the improvement of retinal sensitivity in the area surrounding the CNV. The size of the scotoma decreased with the regression of the CNV after PDT, however, the area of "not seen $0 \mathrm{~dB}$ " was still located in the area demonstrating subretinal fibrosis and chorioretinal atrophy.
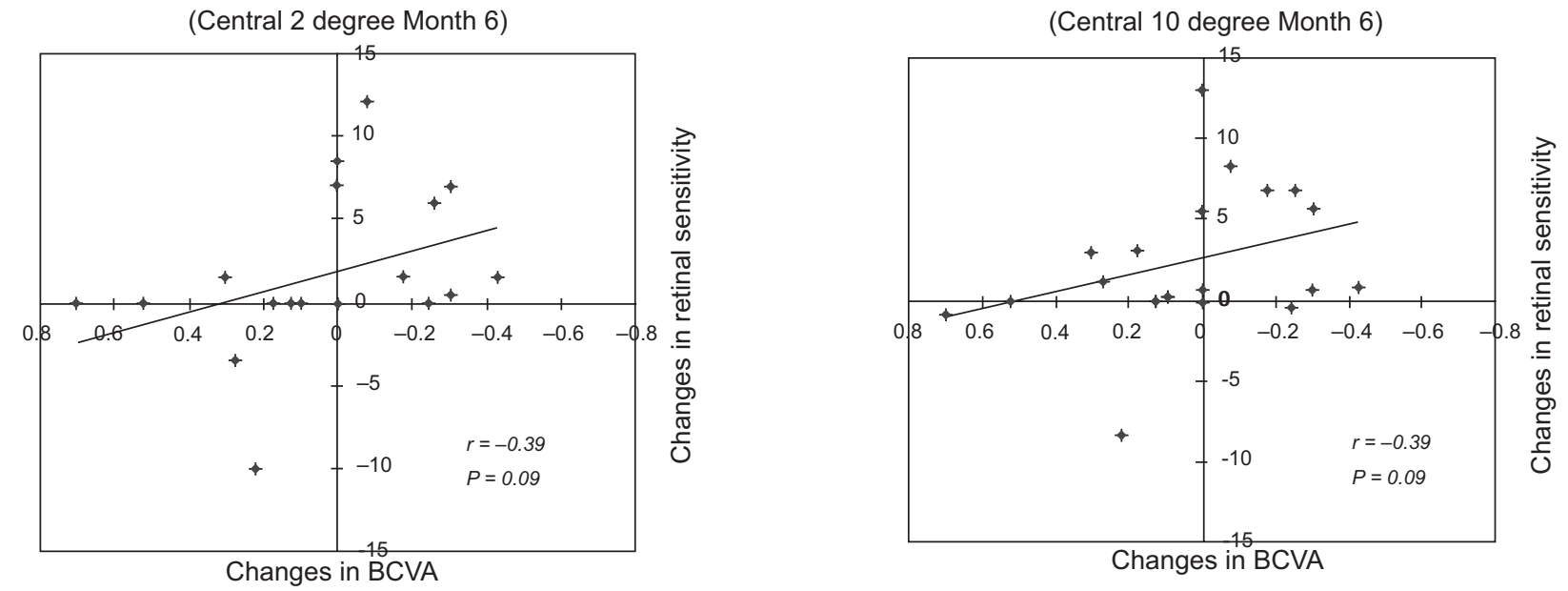

Figure 5 Relationship between changes in retinal sensitivity and changes in the best-corrected visual acuity (BCVA). Changes in retinal sensitivity was not significantly correlated to the BCVA within the central $2^{\circ}(r=-0.39 ; P=0.09$ [Left] $)$ and within the central $10^{\circ}(r=-0.39 ; P=0.09$ [Right]). 
Table 3 Angiographic results and changes in visual acuity and retinal sensitivity at three months after PDT

\begin{tabular}{llll}
\hline & \multicolumn{2}{l}{ Leakages from CNV } & $P$ \\
\cline { 2 - 3 } & $\begin{array}{l}\text { No leakage } \\
(\mathbf{n}=1 \mathbf{1 0 , 5 3 \% )}\end{array}$ & $\begin{array}{l}\text { Others } \\
(\mathbf{n}=\mathbf{9 , 4 7 \% )}\end{array}$ & \\
\hline $\begin{array}{l}\text { Changes in VA } \\
\text { Changes in retinal }\end{array}$ & $-0.09 \pm 0.16$ & $0.15 \pm 0.26$ & 0.04 \\
sensitivity (dB) & & & \\
Within $2^{\circ}$ & $3.9 \pm 5.2$ & $-2.1 \pm 4.1$ & 0.01 \\
Within $10^{\circ}$ & $3.1 \pm 3.4$ & $-0.6 \pm 2.9$ & 0.04 \\
\hline
\end{tabular}

Note: Numerical data were analyzed using Mann-Whitney $U$ test. Abbreviations: CNV, choroidal neovascularization; PDT, photodynamic therapy; $\mathrm{VA}$, visual acuity.

Histologically, CNVs consist mainly of fibrous tissue which is not eliminated by PDT, and the increased subretinal fibrosis probably indicates that the photoreceptors have degenerated. ${ }^{15}$

In conclusion, significant improvements in retinal sensitivities within the central $10^{\circ}$ and the decrease in FT were observed after PDT, although the VA was not significantly improved. A significant correlation was found between the improvement in retinal sensitivity and FT, and between the improvement in FT and BCVA after PDT. Improvements of the visual symptoms were most likely due to the improvements of retinal sensitivity and VA that was cause by the reduced FT after PDT. Retinal sensitivity measured by MP1 may be more sensitive to the changes in macular function because it assesses a larger retinal area than the conventional distant visual acuity. The ability to perform daily tasks is also strongly dependent on the preservation of the central visual field, therefore the retinal sensitivity within $10^{\circ}$ could be a better indicator of practical visual capabilities of patients. Thus, the retinal sensitivities measured by MP1 may be a better way to assess the efficacy of PDT for AMD.

\section{Disclosures}

The authors report no conflicts of interest in this work.

\section{References}

1. Photodynamic therapy of subfoveal choroidal neovascularization in age-related macular degeneration with verteporfin: one-year results of 2 randomized clinical trials-TAP report. Treatment of age-related macular degeneration with photodynamic therapy (TAP) Study Group. Arch Ophthalmol. 1999;117:1329-1345.

Clinical Ophthalmology

\section{Publish your work in this journal}

Clinical Ophthalmology is an international, peer-reviewed journal covering all subspecialties within ophthalmology. Key topics include: Optometry; Visual science; Pharmacology and drug therapy in eye diseases; Basic Sciences; Primary and Secondary eye care; Patient Safety and Quality of Care Improvements. This journal is indexed on
2. Verteporfin In Photodynamic Therapy Study Group. Verteporfin therapy of subfoveal choroidal neovascularization in age-related macular degeneration: two-year results of a randomized clinical trial including lesions with occult with no classic choroidal neovascularizationverteporfin in photodynamic therapy report 2. Am J Ophthalmol. 2001; 131:541-560.

3. Bressler NM; Treatment of Age-Related Macular Degeneration with Photodynamic Therapy (TAP) Study Group. Photodynamic therapy of subfoveal choroidal neovascularization in age-related macular degeneration with verteporfin: two-year results of 2 randomized clinical trials-tap report 2. Arch Ophthalmol. 2001;119:198-207.

4. Chan WM, Lam DS, Lai TY, et al. Photodynamic therapy with verteporfin for symptomatic polypoidal choroidal vasculopathy: oneyear results of a prospective case series. Ophthalmology. 2004;111: $1576-1584$.

5. Sahni J, Stanga P, Wong D, Harding S. Optical coherence tomography in photodynamic therapy for subfoveal choroidal neovascularisation secondary to age related macular degeneration: a cross sectional study. Br J Ophthalmol. 2005;89:316-320.

6. Otani A, Sasahara M, Yodoi Y, et al. Indocyanine green angiography: guided photodynamic therapy for polypoidal choroidal vasuculopathy. Am J Ophthalmol. 2007;144:7-14.

7. Tezel TH, Del Priore LV, Flowers BE, et al. Correlation between scanning laser ophthalmoscope microperimetry and anatomic abnormalities in patients with subfoveal neovascularization. Ophthalmology. 1996;103:1829-1836.

8. Ergun E, Maár N, Radner W, Barbasetto I, Schmidt-Erfurth U, Stur M. Scotoma size and reading speed in patients with subfoveal occult choroidal neovascularization in age-related macular degeneration. Ophthalmology. 2003;110:65-69.

9. Nilsson UL, Frennesson C, Nilsson SE. Patients with AMD and a large absolute central scotoma can be trained successfully to use eccentric viewing as demonstrated in a scanning laser ophthalmoscope. Vision Res. 2003;43:1777-1787.

10. Rohrschneider K, Springer C, Bültmann S, Völcker HE. Microperimetrycomparison between the micro perimeter 1 and scanning laser ophthalmoscope - fundus perimetry. Am J Ophthalmol. 2005;139: 125-134.

11. Yodoi Y, Tsujikawa A, Kameda T, et al. Central retinal sensitivity measured with the micro perimeter 1 after photodynamic therapy for polypoidal choroidal vasculopathy. Am J Ophthalmol. 2007;143: 984-994.

12. Krebs I, Binder S, Stolba U, Brunner S. Reading ability and central visual field after photodynamic therapy. Ophthalmologica. 2004; 218:185-192.

13. Okada K, Yamamoto S, Mizunoya S, Hoshino A, Arai M, Takatsuna Y. Correlation of retinal sensitivity measured with fundus-related microperimetry to visual acuity and retinal thickness in eyes with diabetic macular edema. Eye. 2006;20:805-809.

14. Schmidt-Erfurth UM, Elsner H, Terai N, Benecke A, Dahmen G, Michels SM. Effects of verteporfin therapy on central visual field function. Ophthalmology. 2004;111:931-939.

15. Ghazi NG, Jabbour NM, De La Cruz ZC, Green WR. Clinicopathogenic studies of age-related macular degeneration with classic subfoveal choroidal neovasucularization treated with photodynamic therapy. Retina. 2001;21:478-486.

\section{Dovepress}

PubMed Central and CAS, and is the official journal of The Society of Clinical Ophthalmology (SCO). The manuscript management system is completely online and includes a very quick and fair peer-review system, which is all easy to use. Visit http://www.dovepress.com/ testimonials.php to read real quotes from published authors. 